\title{
New Insight into Electronic Neutrino Creation under X-Ray Absorption by Water Tetrahedron Intercalated with Hydronium Ion $\left(\mathrm{H}_{3} \mathrm{O}^{+}\right)$
}

\author{
Alexander V. Udal'tsov \\ Faculty of Biology, Lomonosov Moscow State University, Moscow 119899, Russia
}

Received: October 09, 2017 / Accepted: October 19, 2017 / Published: November 30, 2017

\begin{abstract}
In contrast to so-called Auger process resulting in electron ejection from oxygen atom of water molecule under X-ray absorption, the coupling of the opposite charges in liquid water leads to polaronic exciton formation. As a result, polaronic exciton stabilized by the structure of water tetrahedron can serve as the reaction center for deuterium creation under X-ray absorption. In this case, X-ray emission spectra of $\mathrm{H}_{2} \mathrm{O}$ and $\mathrm{D}_{2} \mathrm{O}$ give a clue for the deciphering of the events followed by X-ray photon absorption. It appears to be that spin-orbit interaction under proton sharing and the interaction between LO (longitudinal optical) phonon and bipolaron in the excited state are responsible for the energy levels determining neutrino energy. The energy gap between the levels with $p \downarrow \uparrow e$ and $p \downarrow \downarrow e$ spin configurations of proton and electron is found to be $325.51 \mathrm{~cm}^{-1}$ in the case of the spin-orbit interaction in polaronic exciton, while $1,556.054 \mathrm{~cm}^{-1}$ under LO phonon interaction with the proton of the bipolaron producing the change of the nucleus spin direction to the opposite.
\end{abstract}

Key words: Water X-ray emission, polaronic exciton, Compton wavelength, neutrino.

\section{Introduction}

Despite of water is common molecular substance however numerous studies of water during a long time demonstrate many anomalies of the properties that make it the mysterious and puzzle liquid [1-4]. These unusual and unique properties together with the key role of water for humankind attract the attention of many researchers. A major effort to the development of X-ray spectroscopy of water including the measurements under ultra-high vacuum has been made in the last 10 years [5-9]. In addition, the obtained data and the results of the study of so-called Auger process taking place in water under $\mathrm{X}$-ray radiation [5] give

Corresponding author: Alexander V. Udal'tsov, Dr., research fields: (1) chemical engineering of molecular complexes and solid/liquid microcrystals intended for fabrication of photosensitive materials and solar energy conversion; (2) development of polaron and polaronic exciton concepts for description of behavior of small-size protonated water clusters under the interaction with the incident photon. remarkable experimental facts for the explanation of charged particles behavior.

Protonated small-size water clusters including properties of hydronium ion $\left(\mathrm{H}_{3} \mathrm{O}^{+}\right)$are of the interest of many researchers too since the ion in water local structure exhibits characteristic features or vibrational signature [10-13]. It should be noted that such hydronium ions as $\mathrm{H}_{2} \mathrm{DO}^{+}$and $\mathrm{HD}_{2} \mathrm{O}^{+}$characterized by ${ }^{1} \mathrm{H}$ and ${ }^{2} \mathrm{H}$ NMR spectroscopy showed no exchange [14]. The latter means that the $\mathrm{H}_{2} \mathrm{DO}^{+}$and $\mathrm{HD}_{2} \mathrm{O}^{+}$ generated in the case of X-ray absorption by liquid water, which exhibits the emission peaked at 525.88 and $526.6 \mathrm{eV}$, respectively $[9,15]$, represent actually the steps of proton-proton fusion reaction resulting in deuterium creation [15]. While $\mathrm{HD}_{2} \mathrm{O}^{+}$generated in heavy water under X-ray absorption exhibiting the emission at ca. $526.8 \mathrm{eV}$ is formed in the result of the backward reaction. The difference between the maxima of $\mathrm{HD}_{2} \mathrm{O}^{+}$emission in $\mathrm{H}_{2} \mathrm{O}$ and $\mathrm{D}_{2} \mathrm{O}$ that is ca. 
$0.2 \mathrm{eV}$ implies the major energy of the difference disappears in the forward reaction with outgoing neutrino. Thus, the latter is interpreted in terms of deuterium creation under X-ray absorption by liquid water [15], which represents a conceivable explanation of the features of X-ray emission spectra of water and heavy water. Note that a mechanism of deuterium generation should exist since deuterium as an impurity (ca. $0.015 \%$ ) presents in natural water. Proton-proton fusion process is accompanied by pep-neutrino creation, while the backward reaction requires a collision with pep-neutrino [16-18]. In the Sun the process,

$$
p+e^{-}+p \rightarrow d+v_{\mathrm{e}}
$$

occurs at very high temperature but if the same reaction takes place in liquid water pep-neutrino is expected to possess considerably low energy. An estimate of electronic neutrino mass has been suggested in Ref. [15], while the energy required for the proton-proton fusion according to the above reaction is provided by $\mathrm{X}$-ray absorption. In the literature, there are different estimations of neutrino mass like $0.320 \pm 0.081 \mathrm{eV} / \mathrm{c}^{2}$ for the sum of three degenerate neutrinos [18, 19].

Usually the presence of the ionized species in water changes its local structure especially if hole polaron generation can occur that results in hole polaron moving through water together with the self-induced polarization, which can move when proton moves [20, 21]. Hole polaron moving is often accompanied by LO (longitudinal optical) phonons interacting with the self-induced polarization. No doubt, in this case local energy levels in the quasiparticle composed by the electron coupled with proton should exist like those in atomic hydrogen (see below). Then the essential energy characteristics for the process of neutrino creation during the first step of proton-proton fusion reaction resulting in $\mathrm{H}_{2} \mathrm{DO}^{+}$can be evaluated applying polaronic exciton theory. Thus this paper main results of which were presented at XIVth ICMS-2017 in Poland [22], reports further theoretical development in relation to electronic neutrino and deuterium creation under X-ray absorption, which suggests the explanation of neutrino creation in the forward proton-proton fusion reaction.

\section{Theory}

Polaronic exciton theory considers behavior of the quasiparticle under the coupling of the opposite charges $[21,23]$ as the task about hydrogen atom with effective mass $m^{*}$ and charge $e^{*}=e /\left(\varepsilon_{e f}\right)^{1 / 2}$ like for Wannier-Mott exciton [24]. The incident photon interaction with water can be elastic, when photon energy is conserved, or inelastic that usually leads to a decrease of photon energy. The bound state energy $\left(E_{\mathrm{ex}, \mathrm{b}}\right)$ of polaronic exciton can be given by Eq. (1) [21].

$$
\begin{gathered}
E_{\text {ex,b }}=-m_{\mathrm{e}} e^{4} /\left(2 \hbar^{2} n^{2} \varepsilon_{\mathrm{ef}}{ }^{2}\right)\left(m_{\mathrm{r}}{ }^{*} / m_{\mathrm{e}}\right) \\
=-m_{\mathrm{e}} e^{4} /\left(2 \hbar^{2}\right)\left(m_{\mathrm{r}}{ }^{*} / m_{\mathrm{e}}\right)\left(1 / \varepsilon_{\mathrm{ef}}{ }^{2}\right)
\end{gathered}
$$

where $m_{\mathrm{r}}{ }^{*}$ is the reduced effective mass of the exciton, $m_{\mathrm{r}}^{*}=m_{\mathrm{ef}}^{\mathrm{h}} m_{\mathrm{ef}}^{\mathrm{e}} /\left(m_{\text {ef }}^{\mathrm{h}}+m_{\mathrm{ef}}^{\mathrm{e}}\right), m_{\text {ef }}^{\mathrm{h}}$ and $m_{\text {ef }}^{\mathrm{e}}$ are respectively the effective masses of hole and electronic polarons, $e$ and $\hbar$ are the elementary charge $(e=$ $1.6021765 \times 10^{-19} \mathrm{C}$ ) and the reduced Planck constant, $\varepsilon_{\mathrm{ef}}$ is the effective dielectric constant, $\varepsilon_{\mathrm{ef}}{ }^{-1}=1 / \varepsilon_{\infty}-1 / \varepsilon_{\mathrm{s}}$, $\varepsilon_{\mathrm{s}}$ and $\varepsilon_{\infty}$ are the static and high-frequency dielectric constants, respectively. In the latter expression $n=1$ that is omitted since we consider the ground state. In the case of inelastic interactions, the electric field and the energy gap are generated within the area compared with the wavelength of the incident photon. The usage of optical dielectric constant $\left(\varepsilon_{\mathrm{op}}\right)$ instead of $\varepsilon_{\mathrm{ef}}$ is defined by a threshold of kinetic energy evaluated with polaronic exciton radius $\left(R_{\mathrm{ex}}\right)$ in the ground state, which can be obtained with Eq. (3). With $\varepsilon_{\mathrm{op}}=1.333$ of water the calculated $R_{\mathrm{ex}}=1.4813 \AA$ that corresponds to the kinetic energy threshold $E_{\mathrm{k}}=26.11 \mathrm{eV}$ (calculated with Eq. (4)) or wavelength $47.5 \mathrm{~nm}$. So that the local area in liquid water with the polaronic exciton confined inside the area is compared with the wavelength of 47.5 $\mathrm{nm}$. If wavelength of the excitation is less than the threshold, $\varepsilon_{\text {ef }}$ can be replaced by optical dielectric constant $\varepsilon_{\mathrm{op}}=n_{\mathrm{D}}^{2}$ and Eq. (1) can be rewritten as 

Tetrahedron Intercalated with Hydronium lon $\left(\mathrm{H}_{3} \mathrm{O}^{+}\right)$

follows.

$$
\begin{aligned}
E_{\mathrm{ex}, \mathrm{b}} & =-m_{\mathrm{e}} e^{4} /\left(2 \hbar^{2}\right)\left(m_{\mathrm{r}}{ }^{*} / m_{\mathrm{e}}\right)\left(1 / \varepsilon_{\mathrm{op}}{ }^{2}\right) \\
& =-R_{\infty}\left(m_{\mathrm{r}}{ }^{*} / m_{\mathrm{e}}\right)\left(1 / n_{\mathrm{D}}{ }^{4}\right)
\end{aligned}
$$

Effective masses of hole $\left(m_{\text {ef }}^{\mathrm{h}}\right)$ and electronic polarons $\left(m_{\text {ef }}^{\mathrm{e}}\right)$ were assumed as follows, $m_{\text {ef }}^{\mathrm{h}}=10 m_{\mathrm{e}}$ and $m_{\text {ef }}^{\mathrm{e}}=0.5 m_{\mathrm{e}}$ [21], $m_{\mathrm{e}}=9.1093819 \times 10^{-31} \mathrm{~kg}$ that was discussed earlier [21, 25]. In Eq. (2) $m_{\mathrm{e}} e^{4} /\left(2 \hbar^{2}\right)$ is Rydberg constant $\left(R_{\infty}\right), R_{\infty}=13.605692 \mathrm{eV}$. Polaronic exciton radius $\left(R_{e x}\right)$ for the ground state can be defined as the average distance between the hole and electron according to Eq. (3) [21], in which $\varepsilon_{\mathrm{ef}}$ has been replaced by optical dielectric constant.

$$
R_{\mathrm{ex}}=\hbar^{2} /\left(m_{\mathrm{e}} e^{2}\right)\left(m_{\mathrm{e}} / m_{\mathrm{r}}^{*}\right) \varepsilon_{\mathrm{op}}
$$

where, $\hbar^{2} / m_{\mathrm{e}} e^{2}$ is Bohr radius, $r_{\mathrm{B}}=0.5291772 \times 10^{-8}$ $\mathrm{cm}$. In the excited state polaronic exciton has kinetic energy that is equal excitation energy when it absorbs a photon. Thus, kinetic energy $\left(E_{k}\right)$ of polaronic exciton can be defined via momentum $\left(p_{\text {ex }}\right)$.

$$
E_{k}=p_{\mathrm{ex}}{ }^{2} /\left(2 \mathrm{M}_{\mathrm{ef}}\right)
$$

where, $\mathrm{M}_{\mathrm{ef}}$ is polaronic exciton effective mass that is the sum, $\mathrm{M}_{\mathrm{ef}}=m_{\mathrm{ef}}^{\mathrm{h}}+m_{\text {ef }}^{\mathrm{e}}\left(\mathrm{M}_{\mathrm{ef}}=10.5 m_{\mathrm{e}}\right)$ and $p_{\mathrm{ex}}=$ $2 \pi(\hbar / \lambda)$ or $p_{\mathrm{ex}}=\hbar k, k=n\left(2 \pi / l_{\mathrm{ex}}\right)=2 \pi\left(n / l_{\mathrm{ex}}\right)$, where $k$ is wavenumber. The strong coupling between the opposite charges in polaronic exciton allows considering the wavenumber as a combination of four harmonic oscillators with the corresponding $r_{n}$ parameters obtained from the structure being under the consideration and the radii $r_{\mathrm{n}}$ are considered as boundaries of quantum well. Thus, the harmonic approximation gives harmonic mean $r_{\mathrm{ex}}=\left(r_{\mathrm{n}} r_{\mathrm{n}+1}\right)^{1 / 2}$ for each pair of parameters. Fundamental peculiar of liquid water structure is quaternary coordination of water molecules with the formation of the network of hydrogen bonds that implies the existence of four different oscillators under the consideration of polaronic exciton. Thus, the following Eq. (4) has been obtained for kinetic energy of polaronic exciton at harmonic approximation with parameters of four oscillators resulting in the harmonic mean $r_{\mathrm{ex}}[21,23]$.

$$
E_{\mathrm{k}}=\hbar^{2} \mathrm{k}_{\mathrm{ex}}{ }^{2} /\left(2 \mathrm{M}_{\mathrm{ef}}\right)=2 \hbar^{2}\left(2 \pi / r_{\mathrm{ex}}\right)^{2} / \mathrm{M}_{\mathrm{ef}}
$$

It should be noted that the tuning of both $r_{\mathrm{ex}}$ and $\mathrm{M}_{\mathrm{ef}}$ values is impossible in Eq. (4). The former is the result of the combination of four polaronic exciton radii at harmonic approximation and the latter is defined by the effective masses of electronic and hole polarons.

It should be noted that two modes of proton sharing are possible for water that is connected with tetrahedral water molecules coordination in the structure. When an electron of water molecule is in the excited state, the corresponding proton of the same $\mathrm{H}_{2} \mathrm{O}$ can be shared, the energy required for this mode of proton sharing was found to be $E_{\mathrm{v}, \mathrm{sh}}=0.81422 \mathrm{eV}$ [23]. The second mode takes place, when a proton of a neighboring $\mathrm{H}_{2} \mathrm{O}$ molecule is borrowed for the proton sharing as displayed in Scheme 1b. The latter called the kinetic mode of proton sharing, since its energy is defined under the consideration of one-dimensional walk of the electron, requires larger energy, $E_{\mathrm{k}, \mathrm{sh}}=2.55795 \mathrm{eV}$ [15]. It should be noted that the ratio between the energies is $E_{\mathrm{k}, \mathrm{sh}} / E_{\mathrm{v}, \mathrm{sh}}=\pi$ exactly.

As found earlier Compton wavelength $\left(\lambda_{C} / 2 \pi\right.$, $\left.\lambda_{\mathrm{C}}=h /\left(m_{\mathrm{e}} c\right)\right)$, fifteen-step of which is required to obtain polaronic exciton radius providing kinetic energy $534.556 \mathrm{eV}$, plays an important role under inelastic interaction of the incident X-ray photon with water [15]. Tetrahedral water molecule coordination is

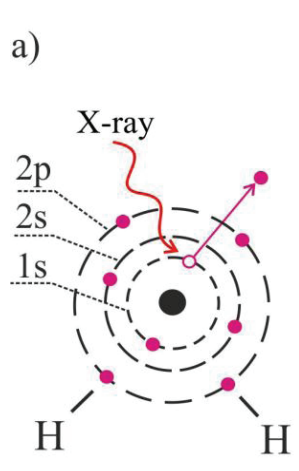

Electron ejection

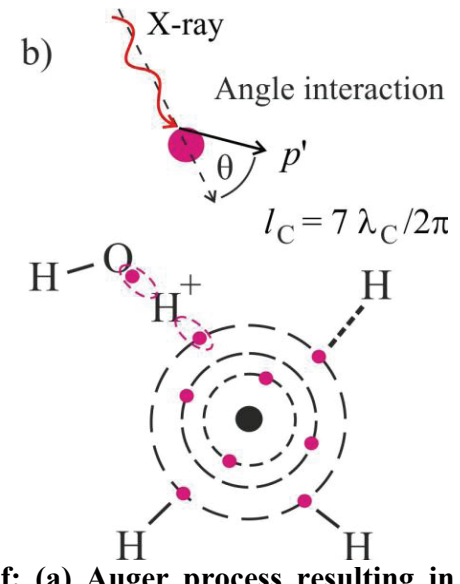

b)
Scheme 1 Illustration of: (a) Auger process resulting in electron ejection; And (b) polaronic exciton generation with strong coupling between the excited electron and the proton borrowed from the neighboring water molecule producing bipolaron. In this case the external electronic shell contains seven electrons, so angular momentum quantum number is $l_{\mathrm{C}}=7$ under the incident photon absorption. 
the consequence of the orbits orientation in water molecule defined by $2 p^{4}$ configuration. Thus, the parameters of polaronic exciton radii in the case of electric field generated by X-ray radiation will be $r_{\mathrm{B}}$, vibration amplitude $a_{\mathrm{x}}$, and the radius of hole polaron $r_{\mathrm{B}} / 2[15,26]$. The most probable wave pathway in the case of polaronic exciton generation is the following, $r_{1} \rightarrow r_{2} \rightarrow r_{1} \rightarrow r_{4}\left(r_{1}=r_{\mathrm{B}}, r_{2}=a_{\mathrm{x}}, r_{4}=r_{\mathrm{B}} / 2\right)$. Therefore, the harmonic mean $r_{\mathrm{ex}, 0}=0.385325 \AA$ is calculated as follows, $r_{\mathrm{ex} 1}=\left(r_{1} \times r_{2}\right)^{1 / 2}=0.5950666 \AA, r_{\mathrm{ex} 2}=\left(r_{\mathrm{ex} 1} \times\right.$ $\left.r_{1}\right)^{1 / 2}=0.5611556 \AA, r_{\mathrm{ex}, 0}=\left(r_{\mathrm{ex} 2} \times r_{\mathrm{B}} / 2\right)^{1 / 2}=0.385325$ $\AA$, where $r_{\mathrm{B}}=0.5291772 \AA$ and $a_{\mathrm{x}}=0.66916 \AA$ [23]. Taking into account $15\left(\lambda_{C} / 2 \pi\right)$ we obtain $r_{\mathrm{ex}}=$ $0.385325-15\left(\lambda_{C} / 2 \pi\right)=0.327401 \AA$.

On the other hand, the same values of polaronic exciton radii can be obtained under consideration of the incident photon interaction with water. The $\theta$ angle between the incident photon direction and the electron momentum directed in the plane of the rotation can therefore assume only the values (see Fig. 1, inset on the right).

$$
\theta=\cos ^{-1}\left\{m /\left[l_{\mathrm{C}}\left(l_{\mathrm{C}}+1\right)\right]^{1 / 2}\right\}
$$

where, $m$ is an integer in the range $7 \geq m \geq-7$ for $l_{\mathrm{C}}=7$ as displayed in Scheme 1. Then, the difference between the neighboring directions of the incident photon and electron momentum expressed as the difference between $\cos \left(\theta_{\mathrm{m}}\right)$ and $\cos \left(\theta_{\mathrm{m}-1}\right)$ is proportional to Compton wavelength and therefore is related to the Compton interaction parameter $\left(r_{\mathrm{C}}\right)$ by the equation.

$$
\begin{gathered}
r_{\mathrm{C}}=\lambda_{\mathrm{C}} /\left[2 \pi\left(\cos \theta_{\mathrm{m}}-\cos \theta_{\mathrm{m}-1}\right)\right]=\beta h /\left(m_{\mathrm{e}} c\right)=\beta \lambda_{\mathrm{C}}(6) \\
\beta=1 /\left[2 \pi\left(\cos \theta_{\mathrm{m}}-\cos \theta_{\mathrm{m}-1}\right)\right]
\end{gathered}
$$

The coefficient of proportionality $\beta$ in Eq. (6) in fact assumes the meaning of the spin-orbit coupling, $\beta=$ 1.19100654 for $l_{\mathrm{C}}=7$. It should be stressed that this parameter takes into account the spin-orbit coupling $\sqrt{ } \beta$ for each particle possessing the spin, i.e. for the electron and the hole both because of the strong coupling between the opposite charges in bipolaron. Then the calculated Compton interaction parameter $r_{\mathrm{C}}$ $=2.8897513 \times 10^{-12} \mathrm{~m}$, which is the constant for the angular momentum quantum number $l_{\mathrm{C}}=7$ with $\beta=$
1.19100654. This approach admits the evaluation of polaronic exciton radii taking into account the interaction angles as follows.

$$
\begin{gathered}
r_{\mathrm{ex}}=r_{\mathrm{ex}, 0}-m_{i} r_{\mathrm{C}}\left(\cos \theta_{\mathrm{m}}-\cos \theta_{\mathrm{m}-1}\right) \\
=r_{\mathrm{ex}, 0}-m_{i} r_{\mathrm{C}} \Delta \cos \theta_{\mathrm{m}}
\end{gathered}
$$

where, $r_{\mathrm{ex}, 0}=0.385325 \AA, \Delta \cos \theta_{\mathrm{m}}$ is invariable within the interval $7 \geq m>-7\left(\Delta \cos \theta_{\mathrm{m}}=0.13363062\right), m_{i}$ is an integer, $1 \leq m_{i} \leq 15$. Thus, the latter relation describes the proportionality between polaronic exciton radii and reduced Compton wavelength found in Ref. [15]. The low spin-orbit coupling parameter $(\beta=1.19100654)$ attests about the weak dependence of polaronic exciton rotation on the quaternary water molecule coordination in the case of X-ray absorption. Fig. 1 shows polaronic exciton radius depending on the interaction angle of the incident photon with water molecule estimated with Eq. (7).

Energy levels between parallel and anti-parallel spin orientations ( $p \downarrow \downarrow e$ and $p \downarrow \uparrow e$ ) of an electron coupled with proton in water should be distanced from each other larger than those in atomic hydrogen in the ground state. Then the ratio of the energy gap $\left(\Delta E_{\mathrm{H}}\right)$ between the levels with different proton and electron spin orientations of hydrogen atom and the gap between the corresponding levels of electron coupled with hole polaron (or the levels splitting, $\Delta E_{\text {spl }}$ ) can be defined via ratio of the frequncies as depicted in Scheme 2.

$$
\hbar \omega_{2} / \hbar \omega_{1}=\Delta E_{\mathrm{sp}} / \Delta E_{\mathrm{H}}
$$

where, $\hbar \omega_{1}$ is the energy corresponding to the frequency measured for hydrogen atom absorbing the wavelength of $21 \mathrm{~cm}$ in the experiment, $f=1,420,405,751.8 \pm$ $0.028 \mathrm{~Hz}$ [27] or $\hbar \omega_{1}=0.0473796359 \mathrm{~cm}^{-1}$. The levels splitting caused by the change of electron spin direction to the opposite in polaronic exciton should be proportional to the ratio of the actual masses of proton and electron $\left(m_{\mathrm{p}} / m_{\mathrm{e}}\right)$, the spin-orbit coupling $\beta=1.19100654$, and the energy ratio obtained for two different modes of proton sharing $\left(E_{\mathrm{k}, \mathrm{sh}} / E_{\mathrm{v}, \mathrm{sh}}=\pi\right)$. The $\left(m_{\mathrm{p}} / m_{\mathrm{e}}\right)$ ratio formally takes into account the following expression $\left(m_{\mathrm{p}} / m^{\mathrm{h}}{ }_{\mathrm{ef}}\right)\left[m_{\mathrm{ef}}^{\mathrm{h}} / m_{\mathrm{e}}\right]$. Then we can write the relation for $\Delta E_{\mathrm{spl}}$ using the frequency of the 

Tetrahedron Intercalated with Hydronium Ion $\left(\mathrm{H}_{3} \mathrm{O}^{+}\right)$

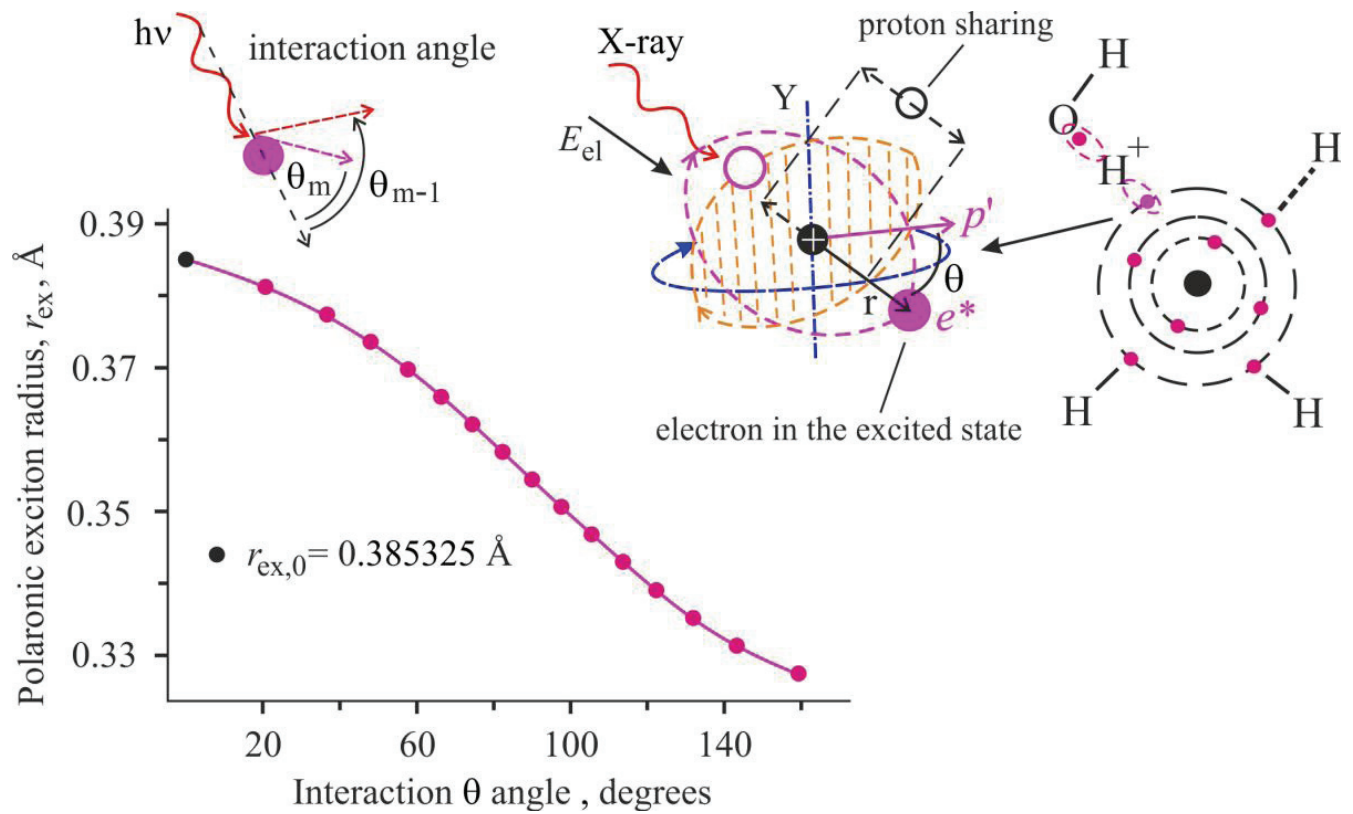

Fig. 1 Plot of polaronic exciton radius $\left(r_{\mathrm{ex}}\right)$ versus interaction $\theta$ angle, the difference $\Delta \cos \theta_{\mathrm{m}}=\cos \theta_{\mathrm{m}}-\cos \theta_{\mathrm{m}-1}=0.13363062$ for $7 \geq m>-7$ (on the left). Inset shows illustration of polaronic exciton generation under the incident $\mathrm{X}$-ray photon interaction with a water molecule that requires $E_{\mathrm{k}, \mathrm{sh}}$ energy (on the right).

Proton and electron spins:

In hole polaron coupled with electron

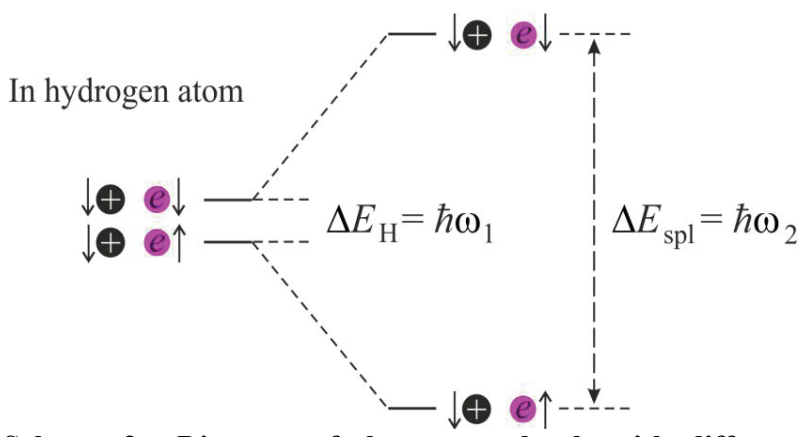

Scheme 2 Diagram of the energy levels with different proton and electron spin orientations depicted for the ground state of atomic hydrogen and the excited state of polaronic exciton due to proton sharing and spin-orbit coupling with $\beta=1.19100654$.

wavelength absorbed by the electron of hydrogen atom.

$$
\Delta E_{\mathrm{spl}}=\hbar \omega_{1}\left(m_{\mathrm{p}} / m_{\mathrm{e}}\right) \beta\left(E_{\mathrm{k}, \mathrm{sh}} / E_{v, \mathrm{sh}}\right)=\pi \Delta E_{\mathrm{H}} \beta\left(m_{\mathrm{p}} / m_{\mathrm{e}}\right)
$$

With $\Delta E_{\mathrm{H}}=\hbar \omega_{1}$ and $m_{\mathrm{p}} / m_{\mathrm{e}}=1,836.15266$ the calculated $\Delta E_{\text {spl }}=325.51014 \mathrm{~cm}^{-1}$ that represents the energy gap arisen between the levels $p \downarrow \uparrow e$ and $p \downarrow \downarrow e$ in polaronic exciton due to spin-orbit coupling under proton sharing.

The frequencies as a doublet at $327,353 \mathrm{~cm}^{-1}$ corresponding to $325.5 \mathrm{~cm}^{-1}$ were observed in resonance Raman spectra of protonated forms of meso-tetraphenylporphine dimer, $\quad \mathrm{TPP}_{2} \mathrm{H}^{+}$and $\left(\mathrm{TPPH}^{+}\right)_{2}$ with a water coating self-organized in the aqueous solutions into nanoparticles and the particles of submicroscopic proportions [21, 28, 29]. This doublet (at $327,353 \mathrm{~cm}^{-1}, \pm 1-2 \mathrm{~cm}^{-1}$ ) is located in the resonance Raman spectra between two doublets of librations at $218,245 \mathrm{~cm}^{-1}$ and $423,447 \mathrm{~cm}^{-1}$ [29] arisen from water molecules connecting the neighboring porphyrin units ( $\mathrm{TPPH}^{+}$and TPP) in the dimer [26]. In the case of spin-orbit interaction with $\beta_{\mathrm{q}}$ $=3.741657387$ depending on the quaternary $\mathrm{H}_{2} \mathrm{O}$ coordination instead of $\beta$, Eq. (8) yields $1,022.6 \mathrm{~cm}^{-1}$ that is found consistent with doublets at 1,000, 1,025 \pm $2 \mathrm{~cm}^{-1}$ observed in the resonance Raman spectra too $[28,29]$. The latter doublet has been assigned to proton sharing in Zundel cation located between the neighboring porphyrin macrocycles of the dimers [26].

\section{Results and Discussion}

3.1 An Estimate of Intermediate Energy Levels Partaking in Electronic Neutrino Creation

Energy levels estimated for liquid water earlier [15], 
which were used for the explanation of X-ray absorption and emission spectra reported in Ref. [9], have the following experimental support. The calculated kinetic energy for gas-phase water $\left(E_{\mathrm{k}}=7.4499\right.$ [23], $\left.166.4 \mathrm{~nm}\right)$ is in a good agreement with the experimental energy of $7.447 \mathrm{eV}$ [30]. The former in the sum with the energy of vibrational mode of proton sharing, $E_{v, \mathrm{sh}}=0.81422 \mathrm{eV}$ [23], which is in consistent with the elastic property of the hydrogen bond, gives the energy $8.26412 \mathrm{eV}$ $(150.0 \mathrm{~nm})$ for the corresponding band of liquid water [23].

Fig. 2 shows X-ray emission and absorption spectra of liquid water. The levels of kinetic energy estimated earlier, so-called pre-edge at $534.98 \mathrm{eV}$ and the main band around ca. $537.85 \mathrm{eV}$, which is the sum of the former and $E_{\mathrm{v}, \mathrm{sh}}$ minus the bound state energy $E_{\text {ex, }}=-2.052 \mathrm{eV}$ calculated with Eq. (2) using $n_{\mathrm{D}}=$ $1.333(534.98157+0.81422-(-2.052)=537.84779$ $\mathrm{eV})$, were obtained applying polaronic exciton theory [15]. New value of $537.86 \mathrm{eV}$ (or 537.86465 if to be more exact) marks this band in the X-ray absorption spectrum in Fig. 2, inset (on the left) because of the activation of hindered water translations $\left(136 \mathrm{~cm}^{-1}\right.$, $0.01686 \mathrm{eV}$ ) participating in deuterium creation too [15]. The realization of the bound state with $E_{\text {ex,b }}$ determines Rydberg energy Ry $=14.018 \mathrm{eV}$ of polaronic exciton as displayed below in Scheme 3a, this energy is accumulated under the X-ray radiation into the pre-edge [15]. The accumulated energy under the excitation defines the emission band around $520.964 \mathrm{eV}(534.98-14.018=520.9636 \mathrm{eV})$ as displayed in Fig. 2 of curve 1. On the other hand, Rydberg energy can be evaluated exactly with the use of spin-orbit interaction parameter $\beta_{\mathrm{q}}=3.741657387$ depending on the quaternary water molecule coordination.

In the case of the kinetic mode of proton sharing $\left(E_{\mathrm{k}, \mathrm{sh}}\right)$ the spin-orbit coupling $\left(\beta_{\mathrm{q}}\right.$ calculated with $l_{\mathrm{C}}=7$ ) can be also defined via the ratio of the Rydberg energy (Ry) and Rydberg constant $\left(R_{\infty}\right)$ taking into consideration the energy $E_{\mathrm{k}, \mathrm{sh}}$ and the features of the tetrahedron structure depicted in Fig. 2, inset (on the right). Then the spin-orbit coupling per the single hydrogen bond can be expressed as follows.

$$
\beta_{\mathrm{q}} / 4=\left(\mathrm{Ry}-E_{\mathrm{k}, \mathrm{sh}} / 2\right) / R_{\infty}
$$

where, $R_{\infty}=13.60569172 \mathrm{eV}$ and Ry is Rydberg energy for polaronic exciton generated in water [15]. Eq. (9) admits to estimate Ry energy exactly with $\beta_{\mathrm{q}}=$ 3.741657387.

$$
\mathrm{Ry}=\beta_{\mathrm{q}} R_{\infty} / 4+E_{\mathrm{k}, \mathrm{sh}} / 2=14.005934 \mathrm{eV} .
$$

The latter is in a good agreement with the earlier obtained $\mathrm{Ry}=14.018 \mathrm{eV}$ (or $14.01753 \mathrm{eV}$ ) evaluated as the accumulated energy [15].

Thus, the accumulated kinetic energy $14.005934 \mathrm{eV}$ provides hole polaron moving through water, the energy of which is the sum of hole polaron self-energy $\left(E_{\mathrm{p}}=1.38 \mathrm{eV}\right.$ [21] or $1.381813 \mathrm{eV}$ exactly) and ionization potential energy $E_{\text {ion }}=12.62 \mathrm{eV}[31]$, a deviation is $0.03 \%$. Therefore, in the ground state hole polaron coupled with the electron can move through water as polaronic exciton. While the hole polaron movement generating LO phonons provides the electron moving via the lowest Franck-Condon states. Earlier kinetic energy for the band at $69 \mathrm{~nm}$ was evaluated as $17.7146 \mathrm{eV}(69.99 \mathrm{~nm})$ under the consideration of the movement of opposite charges coupled in the four-dimension quantum well [23]. The latter implies the electron in such polaronic exciton should move via the lowest Franck-Condon states because of the strong coupling. So that taking into account the energy of the lowest Franck-Condon states $\left(E_{\mathrm{FC}}=0.2445 \mathrm{eV}[21]\right)$ we arrive to the kinetic energy of $17.9591 \mathrm{eV}(69.04 \mathrm{~nm})$.

The generation of polaronic exciton under the excitation into the band ca. $535 \mathrm{eV}(534.98 \mathrm{eV})$ with the accumulation $\mathrm{Ry}=14.005934 \mathrm{eV}$ energy leads to a change of the liquid water structure as mentioned previously [15]. One from the changes consists in that the excitation of the electron of hydronium ion, which can move via the localized states, occurs not from the level of $E_{\mathrm{ex}, \mathrm{g}}=-2.477566 \mathrm{eV}$ but from the lowest 


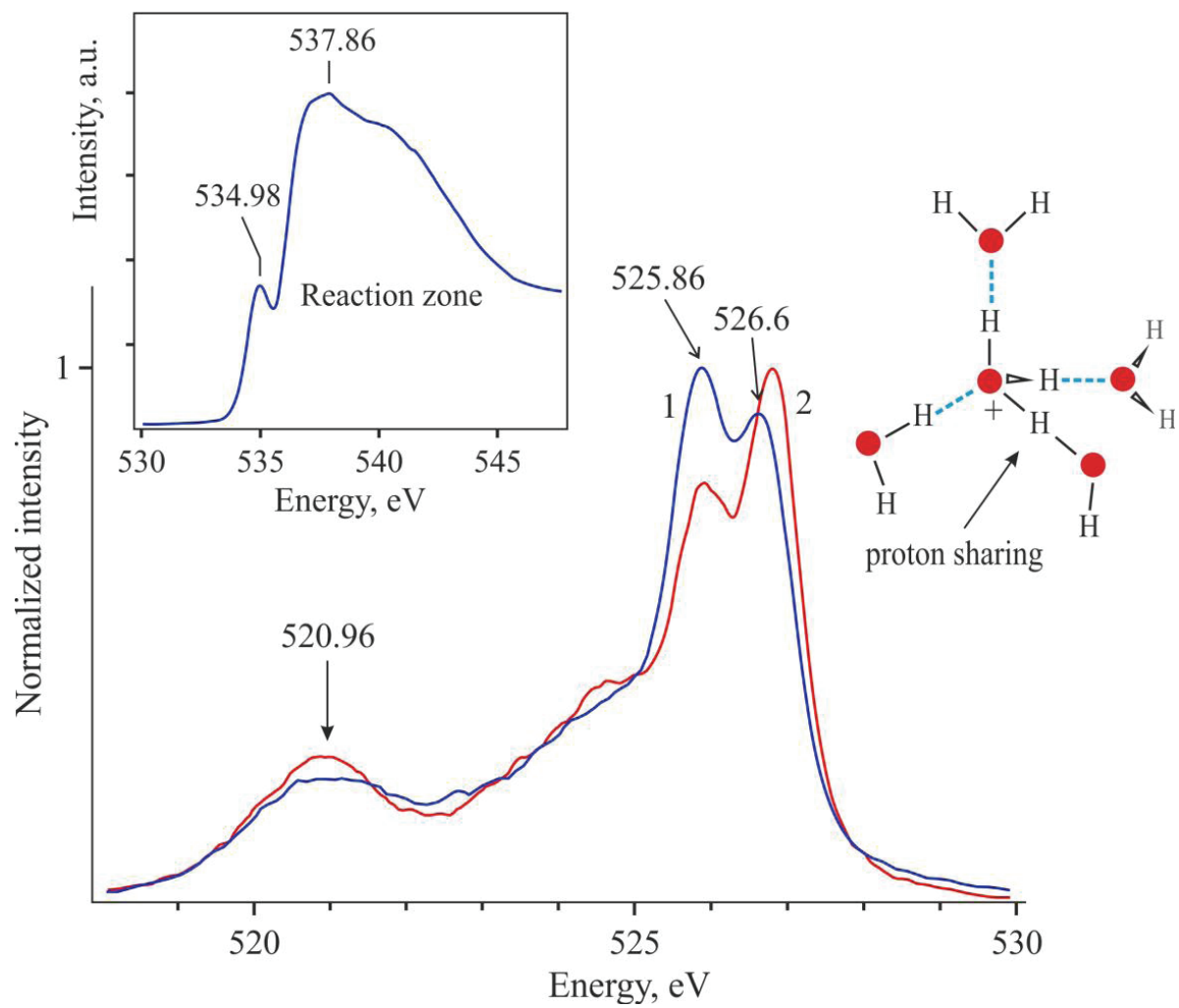

Fig. $2 \mathrm{X}$-ray emission spectra of liquid water, curve 1; and curve 2, $\mathrm{D}_{2} \mathrm{O}$ at $7{ }^{\circ} \mathrm{C}$. Insets show $\mathrm{X}$-ray absorption spectrum of liquid water (on the left), the calculated energies $(\mathrm{eV})$ mark the maxima of the bands; and water tetrahedron intercalated with hydronium ion (on the right). The emission and absorption spectra marked by the calculated energies have been reproduced with Elsevier Science permission from Ref. [15].

a)

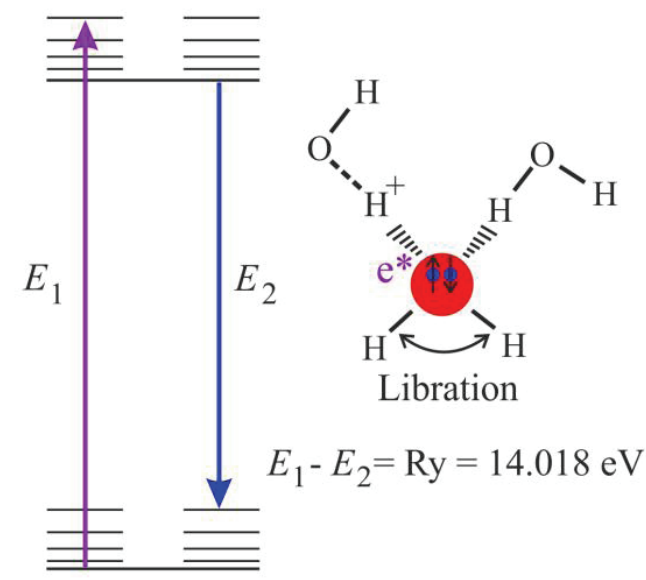

X-ray absorption and emission b)

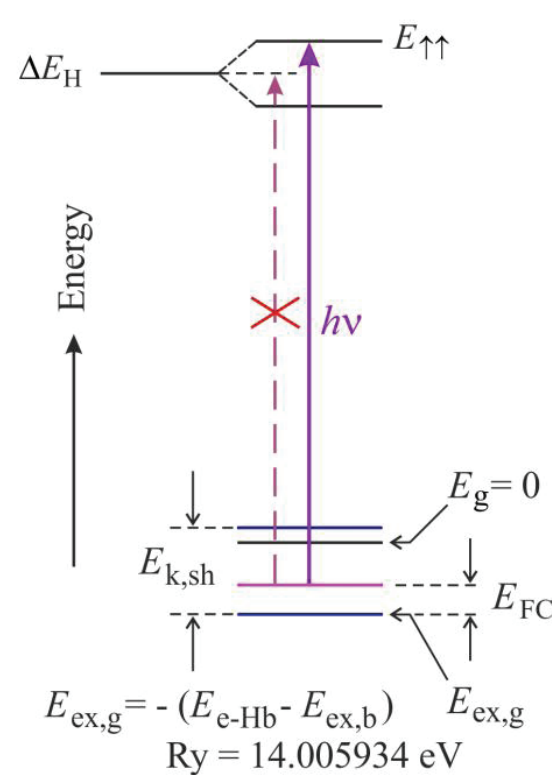

Scheme 3 Illustration of: (a) X-ray energy degradation under inelastic interaction of the incident photon with liquid water that requires Ry energy for polaronic exciton generation, where $E_{1}=534.98157$ and $E_{2}=520.9636 \mathrm{eV}$; And (b) fine structure of the ground and excited states of the tetrahedron interacted with hydronium ion as depicted in Fig. 2, inset (on the right). The lowest Franck-Condon state is higher by $E_{\mathrm{FC}}=0.2445 \mathrm{eV}$ (or more exactly $0.244358 \mathrm{eV}$, see Appendix) relatively to the level of the ground state $E \mathrm{~g}=-2.477566 \mathrm{eV}$. The latter is the sum of $E_{\mathrm{e}-\mathrm{Hb}}=-0.425566 \mathrm{eV}$ and $E_{\mathrm{ex}, \mathrm{b}}=-2.052 \mathrm{eV}$; while the sum $E_{\mathrm{g}}+E_{\mathrm{k}, \mathrm{sh}}$ gives $648 \mathrm{~cm}^{-1}$ that suggests water libration. 
Franck-Condon state as depicted in Scheme $3 \mathrm{~b}$. The latter can take place most likely only in the reaction zone of the absorption spectrum shown in Fig. 2 , inset, where proton-proton fusion reaction can occur [15].

Thus, the emission energy around $525.86 \mathrm{eV}$ is calculated like previously [15] but also taking into account the positive contributions of $\Delta E_{\mathrm{spl}}=0.0403581$ $\mathrm{eV}\left(325.51014 \mathrm{~cm}^{-1}\right)$ and $\hbar \omega_{\mathrm{LO}}=0.368491 \mathrm{eV}$, and the negative contribution of $E_{\mathrm{FC}}=0.244358 \mathrm{eV}$ including neutrino energy $E_{v}$ calculated below. Then the calculated emission is $E_{\mathrm{em}}=525.85876 \mathrm{eV}(537.86465$ $\left.+0.425566+\hbar \omega_{\mathrm{LO}}+\Delta E_{\mathrm{spl}}\right)-(12.3911+0.011923+$ $E_{\mathrm{FC}}+E_{\mathrm{v}}$ ), where $\varepsilon_{\mathrm{st}, \mathrm{D}}=0.011923 \mathrm{eV}$ is the stretch vibration energy of the created deuterium, $\varepsilon_{\mathrm{st}, \mathrm{D}}=$ $136 / \sqrt{ } 2=96.17 \mathrm{~cm}^{-1}[15]$. Scheme 4 illustrates the levels determined by the corresponding inelastic interactions in the excited state.

Scheme 4 assumes the model that LO phonons generating by proton moving through water produces the change of the nucleus spin direction to the opposite under the collision with proton coupled with the electrons in the quasiparticle. As discussed earlier, the excited state of the bipolaron with the energy 537.86 $\mathrm{eV}$ generates deuterium in the proton-proton fusion reaction [15]. This excited state of the bipolaron should have the parallel $\left(\uparrow e \uparrow p e \uparrow^{*}\right)$ spin configuration as displayed in Scheme 4.

\subsection{Consideration of the Events Followed by Polaronic} Exciton Generation in Liquid Water under X-ray Absorption

Assuming the model that the collision of LO phonon moving ahead of the front of the proton results in the change of proton spin direction to the opposite in the bipolaron generated in the excited state, the following events are expected as displayed in Scheme 4. The antiparallel spins of both protons happened in the result of the collision and antiparallel spin of the coupled electron favor the proton-proton fusion reaction, since the electron has been accumulated Rydberg energy under the strong coupling regime in bipolaron [15]. Then we can estimate behavior of the polaronic exciton radius of the electron coupled with the proton for the intermediate spin configuration $\uparrow e \downarrow p e \uparrow^{*}$, while the other electron being in the excited state will emit X-ray quantum $\left(E_{\mathrm{em}}\right)$ in the course of the proton-proton fusion reaction. The angular momentum of the electron operating as electronic polaron with the mass $m_{\text {ef }}^{\mathrm{e}}$ can be written as follows since $m_{\text {ef }}^{\mathrm{e}} \mathrm{v} r=\hbar[l(l+1)]^{1 / 2}$.

$$
2 \pi m_{\mathrm{ef}}^{\mathrm{e}} \omega r_{\mathrm{ex}}{ }^{2}=\hbar[l(l+1)]^{1 / 2}
$$

where $\omega$ is angular frequency and $l$ is the angular momentum quantum number, $l$ is an integer in the range $7 \geq l \geq-7$. This form of Eq. (10) is constrained by the proportionality of $[l(l+1)]^{1 / 2} / 2 \pi=1.19100654$ obtained with the angular momentum quantum number $l=7$ and spin-orbit coupling $\beta=1.19100654$ defined by Eq. (6a). For the angular frequency in Eq. (10) the following relation can be given [15].

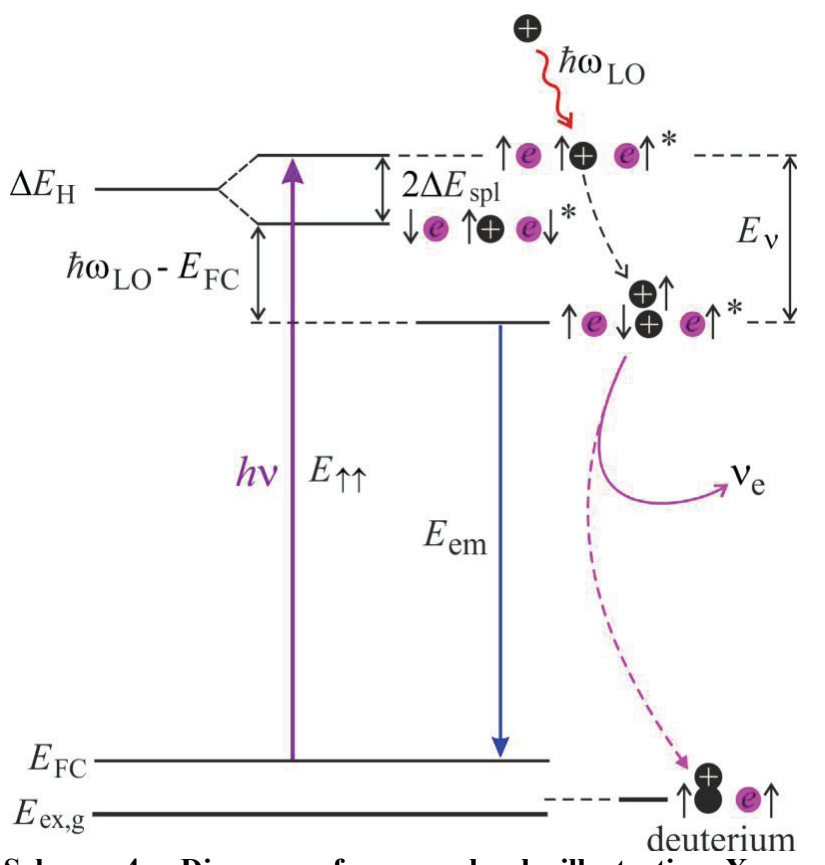

Scheme 4 Diagram of energy levels illustrating X-ray excitation of water tetrahedron intercalated with hydronium ion that is accompanied by the collision of LO phonon with the bipolaron resulting in pep-neutrino creation $\left(v_{\mathrm{e}}\right)$ in the course of proton-proton fusion reaction with the radiative deactivation of the excited state $\left(E_{\mathrm{em}}=\right.$ $525.86 \mathrm{eV}$ ). The events of deuterium creation as $\mathrm{DHO}^{+}$ion occurs in the reaction zone as suggested earlier displayed in Fig. 2, inset, see details in the text. 

Tetrahedron Intercalated with Hydronium Ion $\left(\mathrm{H}_{3} \mathrm{O}^{+}\right)$

$$
\omega=\omega_{\mathrm{a}}+\Delta \omega=\omega_{\mathrm{a}}+\left[2 \Delta E_{\mathrm{k}} /\left(m_{\mathrm{ef}}^{\mathrm{e}} \mathrm{ex}^{2}\right)\right]^{1 / 2}
$$

where, $\Delta E_{\mathrm{k}}$ is the difference of kinetic energy calculated with the decrease of $r_{\mathrm{ex}}$ and $\omega_{\mathrm{a}}$ is the angular frequency obtained under elastic interaction with the incident photon, $\omega_{\mathrm{a}}=1.13105 \times 10^{16} \mathrm{~s}^{-1}[15,23]$. Solving the system of Eqs. (10) and (11) we obtain relations for $r_{\mathrm{ex}}$ denoted as $r_{2}$ and $\omega$.

$$
r_{\mathrm{ex}}=r_{2}=\left[\left(b^{2}+4 a c^{\prime}\right)^{1 / 2}-b\right] /(2 a)
$$

where $a=2 \pi \omega_{\mathrm{a}} m_{\text {ef }}^{\mathrm{e}}=3.2368352 \times 10^{-14} \mathrm{~kg} \cdot \mathrm{s}^{-1}, b=$ $2 \pi\left(2 m_{\text {ef }}^{\mathrm{e}} \Delta E_{\mathrm{k}}\right)^{1 / 2}$, and $c^{\prime}=\hbar[l(l+1)]^{1 / 2}$, and

$$
\omega=\hbar[l(l+1)]^{1 / 2} /\left(2 \pi m^{\mathrm{e}} \mathrm{ef}_{\mathrm{ex}}{ }^{2}\right)
$$

Plots depicted for polaronic radius $r_{2}$ in bipolaron with the spin configuration $\uparrow e \downarrow p e \uparrow^{*}$ and the corresponding angular frequency $(\omega)$ are shown in Fig. 3 , while the inset (on the right) illustrates behavior of the polaronic radius $r_{2}$ in the bipolaron. As we can see from Eq. (10) the expression $\hbar[l(l+1)]^{1 / 2}=0$ when $l=$
0 or $l=-1$, both values imply $r_{2}=0$ as displayed in Fig. 3 , curve 1 (black dots). At the same time, the corresponding angular frequency increases unrestrictedly when $r_{2} \rightarrow 0$ that is depicted out of the range (black dots).

Thus, we can see in Fig. 3 that in the presence of electric $\left(E_{\mathrm{el}}=1.21678 \times 10^{10} \mathrm{~V} / \mathrm{m}\right)$ and magnetic fields [15] generated by the incident photon, the polaronic radius $\left(r_{2}\right.$, curve 1$)$ is rapidly decreased with the growth of the angular frequency (curve 2) that is the prerequisite for proton-proton fusion reaction.

The energy of electronic neutrino can be estimated assuming the model that $\mathrm{LO}$ phonon moving produces the proton spin overturn in the bipolaron under the collision as displayed in Scheme 4 with the use of the energy $\left(\Delta \mathrm{E}_{\mathrm{spl}}\right)$ that is required for the change of electron spin orientation under the excitation. In this

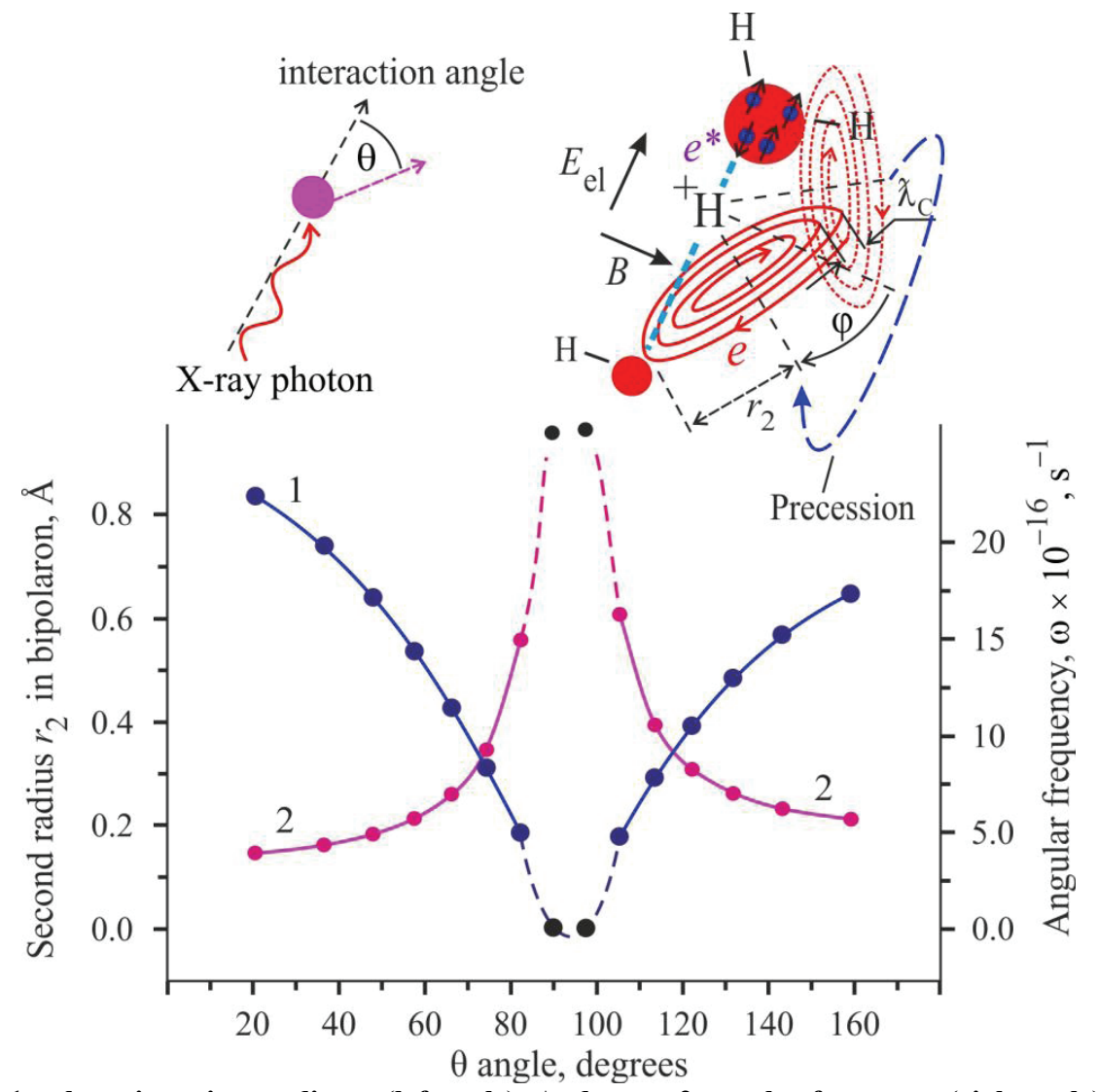

Fig. 3 Plot of curve 1 polaronic exciton radius $r_{2}$ (left scale); And curve 2 angular frequency (right scale) evaluated with Eqs. (12) and (12a) for the electron of the water molecule, proton of which is borrowed for polaronic exciton generation resulting in bipolaron, versus the interaction angle. Insets show illustrations of: Larmor precession of the electron ( $r_{2}$ radius) coupled in bipolaron (on the right); and X-ray photon interaction with electron producing polaronic exciton (on the left). 
case the neutrino energy $\left(E_{v}\right)$ is written taking into account the relation between the energy levels depicted in Scheme 4 and the stretch vibration energy $\left(\varepsilon_{\mathrm{st}, \mathrm{D}}\right)$ of the deuterium created in the course of proton-proton fusion reaction.

$$
E_{\mathrm{v}}=2 \Delta \mathrm{E}_{\mathrm{spl}}+\hbar \omega_{\mathrm{LO}}-E_{\mathrm{FC}}-\varepsilon_{\mathrm{st}, \mathrm{D}}
$$

With $\Delta \mathrm{E}_{\mathrm{spl}}=325.51014 \mathrm{~cm}^{-1}(0.0403581 \mathrm{eV}), \hbar \omega_{\mathrm{LO}}$ $=0.368491 \mathrm{eV}$ (see Appendix), $\varepsilon_{\mathrm{st}, \mathrm{D}}=136 / \sqrt{ } 2=96.17$ $\mathrm{cm}^{-1}(0.011923 \mathrm{eV})$ and $E_{\mathrm{FC}}=0.2445 \mathrm{eV}[21]$ or $E_{\mathrm{FC}}=$ $0.244358 \mathrm{eV}$, the calculated $E_{\mathrm{v}}=0.192784 \mathrm{eV}(1,555$ $\left.\mathrm{cm}^{-1}\right)$ or $0.1929262 \mathrm{eV}\left(1,556.054 \mathrm{~cm}^{-1}\right)$. The latter energy is in a good agreement with the strongly narrowed band observed at $1,556 \mathrm{~cm}^{-1}$ in IR spectra and assigned to neutrino [15].

As known a particle mass is considered in electrodynamics as the coefficient of proportionality between the velocity and the momentum, which can be the sum of mechanic and electromagnetic momenta [32]. In contrast to electron whose electromagnetic mass is $2 / 3 m_{\mathrm{e}}$, the mass of electronic neutrino $3.43026 \times 10^{-37} \mathrm{~kg}[15]$ created in the proton-proton fusion reaction has been found without the mechanic momentum because the mass $3.445467 \times 10^{-37} \mathrm{~kg}$ [22] evaluated using the electron electromagnetic mass is larger by $0.4 \%$. It appears that the absence of the "mechanic" part of neutrino mass provides neutrino specific ability to pass through usual matter unimpeded and undetected.

\section{Conclusions}

The results presented above attest the key role of Compton wavelength $\lambda_{\mathrm{C}} / 2 \pi=r_{\mathrm{B}} \alpha=3.86159264 \times 10^{-13}$ $\mathrm{m}$ that is found under consideration of the incident photon interaction with water in the case of X-ray absorption [15]. The latter generates polaronic exciton, which is stabilized as hydronium ion intercalated into water tetrahedron preventing the local structure from electron ejection like under Auger process. At the same time, the tetrahedron intercalated with $\mathrm{H}_{3} \mathrm{O}^{+}$provides the possibility of deuterium creation under the X-ray absorption due to Rydberg energy (14.005934 eV) accumulation [15]. The energy of outgoing pep-neutrino has been estimated under the consideration of $\mathrm{LO}$ phonon interaction with bipolaron, when LO phonon collides the nucleus producing the change of the nucleus spin direction to the opposite. Thus, Eq. (13) gives an accurate value of neutrino energy $E_{v}=0.192926 \mathrm{eV}\left(1,556.054 \mathrm{~cm}^{-1}\right)$ with the LO phonon energy $\left(\hbar \omega_{\mathrm{LO}}=0.368491 \mathrm{eV}\right)$, Franck-Condon energy $(0.244358 \mathrm{eV})$ and with the use of the energy gap $\left(\Delta E_{\mathrm{spl}}=325.51 \mathrm{~cm}^{-1}\right)$ estimated between the levels with parallel $p \downarrow \downarrow e$ and antiparallel $p \downarrow \uparrow e$ spin configurations.

It should be noted that the energies $\Delta E_{\text {spl }}(325.51014$ $\left.\mathrm{cm}^{-1}\right)$ and $E_{v}\left(1,556 \mathrm{~cm}^{-1}\right)$ characterize fine structure of the quasiparticle composed by the electron coupled with proton in water in the excited state. It appears that the former is proved invariable in dependence on the incident photon energy, X-ray or visible light photon. However, in the case of visible light the former shows up a doublet, i.e. the levels splitting because of polaronic exciton interaction with porphyrin macrocycle $[28,29]$. So that the doublet at 327,353 $\mathrm{cm}^{-1}$ observed earlier in resonance Raman spectra of protonated TPP dimers, which matches $\Delta E_{\mathrm{spl}}=$ $325.51014 \mathrm{~cm}^{-1}$, validates the fine structure of the quasiparticle composed by the electron coupled with proton in water. While the other doublet at 1,000, 1,025 $\mathrm{cm}^{-1}$ observed in the resonance Raman spectra too matches the energy $1,022.6 \mathrm{~cm}^{-1}$ calculated with $\beta_{\mathrm{q}}=3.741657387$ depending on the quaternary $\mathrm{H}_{2} \mathrm{O}$ coordination. Therefore, this doublet validates the spin-orbit coupling combined with the proton sharing between the central and the surrounding oxygen atoms of the tetrahedron. Note that similar coupling parameter of 3.7182 is found previously under the description of ESR spectra broadening due to spin-spin interaction between manganese aquacomplexes coupled via hydrogen bonding [33].

\section{Appendix}

The energy of LO phonons $\left(\hbar \omega_{L O}=0.368491 \mathrm{eV}\right)$ 


\section{New Insight into Electronic Neutrino Creation under X-Ray Absorption by Water Tetrahedron Intercalated with Hydronium Ion $\left(\mathrm{H}_{3} \mathrm{O}^{+}\right)$}

has been evaluated using aqueous solutions of mono-protonated meso-tetraphenylporphine dimer $\left(\mathrm{TPP}_{2} \mathrm{H}^{+}\right)$, electronic spectrum of which is interpreted as LO phonons originated due to proton movement through the solid matrix [25]. The previous estimate has been made with the use of base line (Fig. 4, curve 2), which gives a plane maximum around ca. $533 \mathrm{~nm}$ (curve 3 ). In contrast, the other mode of the maximum location displayed in Fig. 4, curve 4 gave the band somewhat peaked at $535.5 \mathrm{~nm}$. The latter corresponds to $\hbar \omega_{\mathrm{LO}}=0.368491 \mathrm{eV}$ that is the most appropriate estimation.

It was found that the energy of the lowest Franck-Condon states, which is calculated with Eq. (14) $[20,21]$, is independent from Fröhlich coupling constant in the range between 4.2 and 4.6 as displayed in Fig. 5. At the same time experimental values [21] are obeyed the theoretical dependence shown in Fig. 5, curve 1 .

$$
E_{\mathrm{FC}}=\left[\alpha^{2} /(9 \pi)\right] \hbar \omega_{\mathrm{LO}}
$$

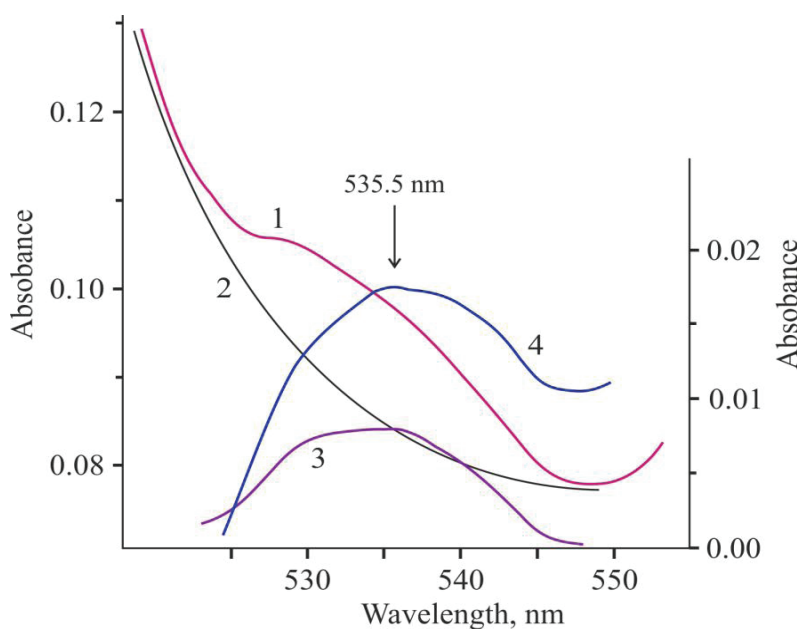

Fig. 4 Electronic (1) and difference $(3,4)$ absorption spectra of protonated TPP dimers in aqueous glycerol with 24 mol. $\mathrm{L}^{-1}$ water and $0.74 \mathrm{~mol} \cdot \mathrm{L}^{-1} \mathrm{THF}$ in the presence of $0.4 \mathrm{~N} \mathrm{HCl}$ at $77 \mathrm{~K}$ : (3) curve 1 minus the base line (curve 2) that is the wing of the band around $467 \mathrm{~nm}$ (left scale); and (4) curve 1 minus the spectrum of the same sample but at $293 \mathrm{~K}$ (right scale), which was obtained without a shoulder at approximately $533-535 \mathrm{~nm}$.

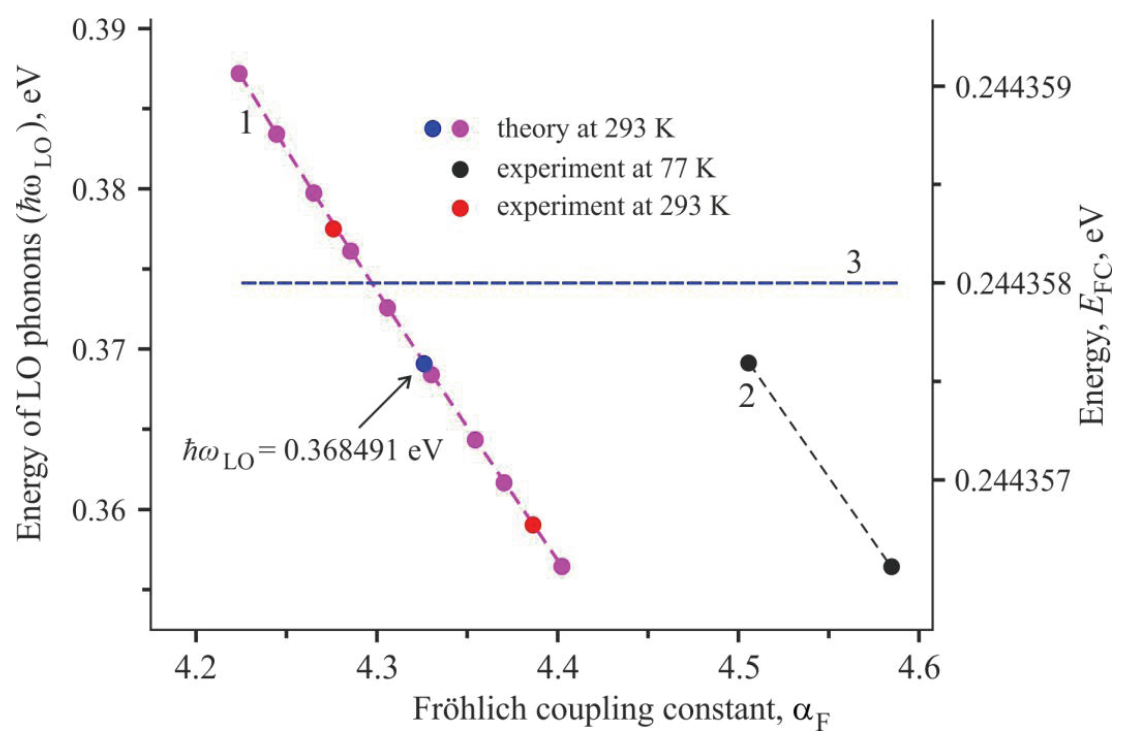

Fig. 5 Plot of curve 1 theoretical energy of LO phonons and curves 1 and 2 the experimental energies (left scale); And curve 3 the energy of the lowest Franck-Condon transitions evaluated with Eq. (14) (right scale) versus Fröhlich coupling constant. 
Thus, the energy of the lowest Franck-Condon states is found invariable within 4.2-4.6 values of Fröhlich coupling constant that is $E_{\mathrm{FC}}=0.244358 \mathrm{eV}$.

\section{References}

[1] Bernal, J. D. 1959. “The Structure of Liquids.” Proc. Roy. Inst. Gr. Brit. 37: 355-83.

[2] Yukhnevich, G. V. 1973. Infrared Spectroscopy of Water. Nauka, Moscow, Russian.

[3] Eisenbeerg, D., and Kauzmann, W. 1969. The Structure and Properties of Water. Oxford: Oxford University Press.

[4] Franks, F. (ed.) 1979. Water, a Comprehensive Treatise. New York: Plenum Press, V.6.

[5] Nordlund, D., Ogasawara, H., Bluhm, H., Takahashi, O., Odelius, M., Nagasono, M., Pettersson, L. G. M., and Nilsson, A. 2007. "Probing the Electron Delocalization in Liquid Water and Ice at Attosecond Time Scales." Physical Review Letters 99 (21): 217406-9.

[6] Nordlund, D., Odelius, M., Bluhm, H., Ogasawara, H., Pettersson, L. G. M., and Nilsson, A. 2008. "Electronic Structure Effects in Liquid Water Studied by Photoelectron Spectroscopy and Density Functional Theory." Chemical Physics Letters 460 (1-3): 86-92.

[7] Tokushima, T., Harada, Y., Takahashi, O., Senba, Y., Ohashi, H., Pettersson, L. G. M., Nilsson, A., and Shin, S. 2008. "High Resolution X-Ray Emission Spectroscopy of Liquid Water: The Observation of Two Structural Motifs." Chemical Physics Letters 460 (4-6): 387-400.

[8] Huang, C., Wikfeldt, K. T., Tokushima, T., Nordlund, D., Harada, Y., Bergmann, U., Niebuhr, M., Weiss, T. M., Horikawa, Y., Leetmaa, M., Ljungberg, M. P., Takahashi, O., Lenz, A., Ojamae, L., Lyubartsev, A. P., Shin, S., Pettersson, L. G. M., and Nilsson, A. 2009. "The Inhomogeneous Structure of Water at Ambient Conditions." Proc. Nat. Acad. Sci. 106 (36): 15214-8.

[9] Nilsson, A., and Pettersson, L. G. M. 2011. "Perspective on the Structure of Liquid Water." Chemical Physics 389 (1-3): 1-34.

[10] Bakker, H. J., and Nienhuys, H.-K. 2002. "Delocalization of Proton in Liquid Water.” Science 297 (5581): 587-90.

[11] Headrick, J. M., Diken, E. G., Walters, R. S., Hammer, N. I., Christie, R. A., Cui, J., Myshakin, E. M., Duncan, M. A., Johnson, M. A., and Jordan, K. D. 2005. "Spectral Signatures of Hydrated Proton Vibrations in Water Clusters." Science 308 (5729): 1765-9.

[12] Marcus, Y. 2012. "Volumes of Aqueous Hydrogen and Hydroxide Ions at 0 to $200{ }^{\circ}$ C." J. Chem. Phys. 137 (15): 154501-5.

[13] Kulig, W., and Agmon, N. 2014. "Both Zundel and Eigen Isomers Contribute to the IR Spectrum of the Gas-Phase
$\mathrm{H}_{9} \mathrm{O}_{4}{ }^{+}$Cluster." J. Phys. Chem. B 118 (1): 278-86.

[14] Olah, G. A. 2001. A Life of Magic Chemistry: Autobiographical Reflections of a Nobel Prize Winner. New York: Wiley Interscience.

[15] Udal'tsov, A. V. 2017. "Water Tetrahedron Intercalated with Hydronium Ion $\left(\mathrm{H}_{3} \mathrm{O}^{+}\right)$as Molecular Reactor Producing Deuterium under X-Ray Absorption." J. Mol. Liquid 237: 99-107.

[16] Bahcall, J. N. 1989. Neutrino Astrophysics. Cambridge Univ. Press.

[17] Bellini, G., Benziger, J., Bick, D., et al. 2012. "First Evidence of Pep Solar Neutrinos by Direct Detection in Borexino (Borexino Collaboration)." Phys. Rev. Lett. 108: 51302-7.

[18] Lesgourgues, J., Mangano, G., Miele, G., and Pastor, S. 2013. Neutrino Cosmology. Cambridge Univ. Press.

[19] Battye, R. A., and Moss, A. 2014. "Evidence for Massive Neutrinos from Cosmic Microwave Background and Lensing Observations." Phys. Rev. Lett. 112: 51303-7.

[20] Devreese, J. T. 1996. "Polarons, in: Encyclopedia of Applied Physics." G. L. Trigg (ed.), VCH, Weinheim, 14: 383-409.

[21] Udal'tsov, A. V. 2016. "Polaronic Exciton in Self-Organized Assemblies of Protonated Meso-tetraphenylporphine Dimers and Water at room Temperature." J. Mol. Struct. 1125: 522-31.

[22] Udal'tsov, A. V. 2017. "Water Tetrahedron Intercalated with Hydronium Ion $\left(\mathrm{H}_{3} \mathrm{O}^{+}\right)$as Molecular Reactor Producing Deuterium under X-ray Absorption: New Insight, XIVth International Conference on Molecular Spectroscopy." Krakow-Bialka Tatrzanska, 3-7 September 2017, Abstracts, p.119.

[23] Udal'tsov, A. V. 2017. "Polaronic Exciton and Its Energy Levels in Water Structure.” J. Mol. Liquid 227: 262-7.

[24] Anselm, A. I. 1978. "Introduction in Semiconductors Theory" Nauka, Moscow, (Russian).

[25] Udal'tsov, A. V. 2015. "Hole Polaron of Small Radius in Assemblies of Hydrated Mono-Protonated Meso-tetraphenylporphine Dimers at 77 K." J. Phys. Chem. Sol. 86: 162-9.

[26] Udal'tsov, A. V., Bolshakova, A. V., and Vos, J. G. 2015. "The Role of Zundel-Like Ions in the Supramolecular Self-organization of Porphyrin Assemblies." J. Mol. Struct. 1080: 14-23.

[27] Feynman, R. P., Leighton, R. B., and Sands, M. 1966. "The Feynman Lectures on Physics." Mir, Moscow, V. 8 (Russian).

[28] Udal'tsov, A. V., Bolshakova, A. V., and Vos, J. G. 2014. "Highly Ordered Surface Structure of Large-Scale Porphyrin Aggregates Assembled from Protonated TPP and Water." J. Mol. Struct 1065-6: 170-8.

[29] Udal'tsov, A. V., and Churin, A. A. 1998. "Molecular 


\section{Tetrahedron Intercalated with Hydronium Ion $\left(\mathrm{H}_{3} \mathrm{O}^{+}\right)$}

Complexes between Dimeric forms of Porphyrin and Water and Their Vibrational Dynamics." 17th IUPAC Symposium on Photochemistry, Virtual Session, Internet Photochem. Photobiol. http://www.photobiology.com/ IUPAC98/ Udaltsov/index.htm.

[30] Mota, R., Parafita, R., Giuliani, A., Hubin-Franskin, M.-J., Lourenço, J. M. C., Garcia, G., Hoffmann, S. V., Mason, N. J., Ribeiro, P. A., Raposo, M., and Limão-Vieira, P. 2005. "Water VUV Electronic State Spectroscopy by Synchrotron Radiation." Chem. Phys. Lett. 416: 152-9.

[31] Wozniak, B., and Dera, J. 2007. Atmospheric and
Oceanographic Sciences Library. New York: Springer, Chap. 2.

[32] Feynman, R. P., Leighton, R. B., and Sands, M. 1966. "The Feynman Lectures on Physics." Mir, Moscow, V. 6 (Russian).

[33] Udal'tsov, A. V., Lyutoev, V. P., and Belykh, D. V. 2016. "Hydrogen Bonding in Assemblies of $\mathrm{Mn}(\mathrm{II}) / \mathrm{Mn}(\mathrm{III})-\mathrm{NEt}_{3}$ Complex Self-organized with the Formation of Hydrophobic Core." Key Engineer. Mater. 708: 103-9. DOI: 10.4028/www.scientific.net/KEM. 708.103 . 